\title{
Actividad comercial y uso del espacio público en los centros urbanos: un análisis clúster
}

\author{
Àngels Pérez Mateos \\ Àngel Cebollada Frontera \\ Ana Vera Martín \\ Universitat Autònoma de Barcelona \\ angels.perez@uab.cat \\ angel.cebollada@uab.cat \\ ana.vera@uab.cat
}

\section{Resumen}

La relación entre espacio público y actividad comercial es constitutiva en los centros urbanos. De este modo, los establecimientos se concentran en espacios urbanos de mayor atracción (calles comerciales, centros comerciales y mercados) y también forman parte de los procesos socioespaciales de dichos entornos. El objetivo de este artículo es analizar el papel de la actividad comercial en el uso del espacio público basándonos en la dimensión humana del urbanista Jan Gehl (2010). Para ello se utilizan las variables del censo de la actividad comercial, flujos de peatones y la caracterización física de las calles de los siete centros urbanos trabajados. De esta manera, se obtendrán resultados del análisis clúster que evidencian las múltiples relaciones entre los distintos usos comerciales, los rasgos de las calles y su espacio público y el paso diario de peatones, así como la naturaleza independiente y explicativa que ha mostrado esta última variable en el análisis.

Palabras clave: análisis clúster; centros urbanos; actividad comercial; flujo de peatones

Resum. Activitat comercial i ús de l'espai públic als centres urbans: una anàlisi clúster

La relació entre espai públic i activitat comercial és constitutiva als centres urbans. D’aquesta manera, els establiments es concentren en espais urbans amb més atracció (carrers comercials, centres comercials i mercats) i també formen part dels processos socioespacials d'aquests entorns. L'objectiu d'aquest article és analitzar el paper de l'activitat comercial en l'ús de l'espai públic basant-nos en la dimensió humana de l'urbanista Jan Gehl (2010). Per fer-ho s'utilitzen les variables del cens de l'activitat comercial, fluxos de vianants i la caracterització física dels carrers dels set centres urbans treballats. D'aquesta manera s'obtindran resultats de l'anàlisi clúster que evidencien les múltiples relacions entre els diferents usos comercials, els trets dels carrers i el seu espai públic i el pas diari de vianants, així com la naturalesa independent $i$ explicativa que ha mostrat aquesta darrera variable en l'anàlisi.

Paraules clau: anàlisi clúster; centres urbans; activitat comercial; flux de vianants 
Résumé. Active commerciale et usage de l'espace public dans les centres urbains : une analyse de cluster

La relation entre l'espace public et l'activité commerciale est constitutive des centres urbains. Ainsi, les établissements sont concentrés dans des espaces urbains plus attractifs (rues commerçantes, centres commerciaux et marchés) et font également partie des processus socio-spatiaux de ces environnements. L'objectif de cet article est d'analyser le rôle de l'activité commerciale, des flux de piétons et de la caractérisation physique des rues des sept centres urbains étudiés. L'étude de ces variables permet d'obtenir les résultats de l'analyse par grappes, qui montrent les multiples relations entre les différents usages commerciaux, les caractéristiques des rues et de leur espace public et du passage piéton quotidien, ainsi que le caractère indépendant et explicatif que cette dernière variable a montré dans l'analyse.

Mots-clés: analyse des grappes; centres urbains; activité commerciale; flux piétons

\section{Abstract. Commercial activity and use of public space in urban centres: a cluster analysis}

The relationship between public space and commercial activity is essential in urban centres. Thus, establishments are concentrated in urban spaces of greater attraction (shopping streets, shopping centres and markets) and are also part of the socio-spatial processes of these environments. The objective of this article is to analyse the role of commercial activity in the use of public space based on the human dimension of the urban planner Jan Gehl (2010). For this, the variables of the census of commercial activity, pedestrian flows and the physical characterization of the streets of the seven urban centres studied are used. Thus, the results of the cluster analysis will be obtained to show the multiple relationships between the different commercial uses, the features of the streets and their public space and daily pedestrian flow, as well as the independent and explanatory nature that this last variable has shown in the analysis.

Keywords: cluster analysis; urban centres; commercial activity; pedestrian flow

\section{Sumario}

\section{Introducción}

2. Marco teórico: los centros urbanos y la actividad comercial

3. Metodología y datos

4. Resultados: clústeres de las calles de las ciudades medianas
5. Discusión: la frecuentación del espacio público y su organización comercial

6. Consideraciones finales

Referencias bibliográficas

\section{Introducción}

Los centros urbanos de las ciudades son el espejo de los cambios sociales, económicos y culturales tanto en su época de esplendor como en su época de decadencia. Allí es donde tradicionalmente se ha concentrado la actividad comercial en combinación con los servicios a la sociedad (Espinosa, 2003, 2007; Rivas, 2007) y, al mismo tiempo, donde se han llevado a cabo prácticas 
con el fin de peatonalizar el espacio público para conseguir entornos más animados, sostenibles y saludables (Gehl, 2010).

La relación entre ciudad y comercio se basa en obtener una máxima rentabilidad económica por parte de los comerciantes y una ventaja comparativa para la ciudad. La actividad comercial se localiza en aquellos espacios donde se encuentran economías de aglomeración (Fujita y Thiesse, 2002; Fujita y Krugman, 2004; Krugman, 1991; O'Sullivan, 2007).

Inicialmente, dichas economías se utilizaban para analizar la actividad industrial (Marshall, 1920; Porter, 1990), pero posteriormente se desarrollaron estudios relacionados con la concentración de empresas de servicios orientadas a la producción (Daniels, 1985) o la organización del espacio comercial según la tipología comercial (Londoño, 2015; Sevtsuk, 2020).

El origen de las economías de aglomeración puede ser diverso (Rosenthal y Strange, 2004). Se contemplan desde ventajas naturales — por la proximidad y el fácil acceso al centro de negocios de la ciudad, más que al resto del área urbana, o características físicas del entorno-; factores externos - como el mercado de trabajo, categorías comerciales que muestran patrones de distribución espacial distintos entre sí tomando de referencia elementos simbólicos, lugares públicos altamente frecuentados (Zhang et al., 2019)—; patrones espaciales comerciales según horario de apertura (Mulícêk y Osman, 2018), hasta el azar (Krugman, 1991). En la última década, asistimos al declive de determinados modelos de aglomeración comercial (las grandes superficies periurbanas) no solo por la irrupción del comercio online, sino porque han perdido competitividad frente a un sector tradicional reorganizado para operar como un centro comercial urbano en abierto, como los ya consolidados BIDs (business improvement districts) en Europa (Hamburgo, Berlín, Londres, Edimburgo), en Canadá (Toronto), en EE. UU. (Los Ángeles y San Francisco), entre otros, o también las recién aprobadas en Cataluña APEUs (áreas de promoción económica y urbana). Así, el discurso de los bajos costes y la eficiencia del tamaño que hizo proliferar las grandes superficies comerciales periurbanas en los noventa (Bratos, 2013) no tendrían cabida en la transición a la sostenibilidad. El «nuevo» modelo de centro comercial urbano lo supera en términos de inclusión social, ya sea por el carácter excluyente y exclusivo de muchas grandes superficies (Sassano, 2015) o por negar, en algunos casos, a las rentas más bajas el acceso a productos alimentarios de mayor calidad, como apuntan Guy y David (2004). En términos económicos, el «nuevo» modelo prioriza el desarrollo local (beneficios que revierten localmente y de distintas formas), junto con la atracción de servicios culturales frente al monopolio de las grandes firmas y cadenas. Por último, en términos socioambientales, fomenta la movilidad activa y contribuye a reducir el impacto de la emisión de $\mathrm{CO}_{2}$, también con el fomento del consumo responsable frente al compulsivo y ocioso que favorecen los grandes centros (Sassano, 2015; Casares, 1995).

El objetivo principal es analizar el papel de la actividad comercial en el uso del espacio público en los centros comerciales urbanos de siete ciudades medianas catalanas. Se plantea relacionar la actividad comercial con la presencia de 
equipamientos municipales y con la calidad del espacio público, según la oferta de movilidad y sus características físicas y el flujo de peatones.

No hay un único método para explicar la concentración espacial de las actividades económicas. Una forma de hacerlo es según el tipo de variable utilizada, y es así como Duranton y Overman (2005) identifican tres grupos metodológicos que posteriormente Garrocho et al. (2012) agrupan en dos. Por un lado, se encuentran los métodos que utilizan variables espaciales o variables espaciales discretas, como por ejemplo el índice de Gini o cálculos estadísticos que tan solo se asocian a un territorio porque los datos están organizados en unidades geográficas (países, regiones, municipios) (Krugman, 1991). Por otro lado, están aquellos análisis donde las variables hacen referencia a espacios continuos, y se realizan cálculos como el del vecino más próximo, análisis de patrones de puntos en espacios continuos (Anselin, 1992, 1996) o la K de Ripley (1976).

En este contexto, se utiliza la metodología del análisis clúster porque se trata de un espacio continuo y permite identificar categorías de calles que responden a preguntas como las siguientes: ¿cómo son las calles que registran más afluencia diaria de personas?, ¿qué tipos de usos concentran?, ¿con qué elementos artificiales o naturales cuentan para atraer a peatones?, ¿es posible distinguir patrones de comportamiento similares entre calles tan distanciadas geográficamente?

La hipótesis principal es que los centros urbanos son, generalmente, puntos de concurrencia de personas donde se concentra el mayor número de locales comerciales que, aun perteneciendo a distintas ciudades, tienden a experimentar dinámicas similares entre sí, tanto en lo relativo al paisaje urbano como en lo que se refiere a la afluencia de personas. Para ello, se tienen en cuenta las distintas tipologías de actividad comercial y de servicios, los atributos de su espacio público, el hecho de que por naturaleza sean más o menos soleados, de trama más o menos irregular y con el mayor número posible de accesos locales o interurbanos, y se establecen como claves en las dinámicas.

Para demostrarlo, se utilizan como área de estudio los centros urbanos de siete ciudades medianas catalanas (figura 1) que forman parte de la Fundació Comerç Ciutadà, la fuente principal de datos en esta investigación.

Se trata de demarcaciones que demográficamente suponen el $11 \%$ de los habitantes de Cataluña (2019) y funcionalmente preservan la centralidad de sus elementos administrativos y culturales. Además, articulan un territorio mucho más amplio que el de su propio límite, ya que son cabeceras de comarca, por lo que resultan áreas comerciales dotadas para cubrir la demanda de bienes y servicios de todos los municipios de sus áreas de influencia.

La unidad de análisis es la calle —un total de 82 calles (inicialmente 111) en centros urbanos y núcleos históricos-, que coincide con las áreas de peatones y con espacios con distintos grados de restricción a la circulación rodada motorizada. Por otra parte, son centros que han evolucionado y se han revitalizado a distintos ritmos, en general, sin alcanzar un grado extremo de desertización comercial por la pérdida de diversidad social y funcional, característica de otras ciudades catalanas y españolas (Zárate, 2006; Segado y Espinosa, 2015). 
Figura 1. Mapa de las áreas de estudio

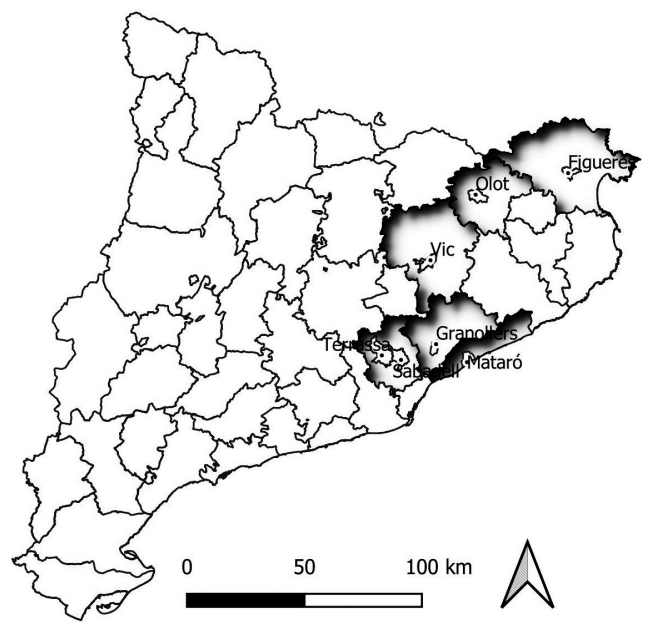

Fuente: elaboración propia con las bases del ICGC y el sistema de información geográfica QGIS.

\section{Marco teórico: los centros urbanos y la actividad comercial}

El comercio ha sido desde siempre una de las principales actividades económicas desarrolladas en el centro de las ciudades. La presencia de comercio en los espacios públicos urbanos (mercados o mercadillos) o en las plantas bajas de los edificios ha caracterizado el paisaje de los centros urbanos (Ferrer y Carrera, 1995) y ha ejercido un papel fundamental en el proceso de urbanización. De hecho, Isenberg (2004) presenta la actividad comercial como un elemento clave para explicar la diversidad social, económica y étnica que caracterizaba el bullicio de los centros urbanos de las ciudades americanas de principios del siglo xx. Los cambios en las prioridades de la sociedad llevaron a desplazar la actividad comercial hacia las áreas suburbanas, donde se priorizaba el acceso a través de una vía segregada con espacio suficiente para aparcar el automóvil. Este es el caso de los centros comerciales suburbanos surgidos en los años cincuenta en las ciudades americanas de Detroit o Baltimore. En las ciudades españolas también se reprodujo este proceso de creación de nuevas centralidades comerciales alejadas de los centros urbanos, pero no fue hasta los años ochenta cuando se establecieron los primeros centros comerciales en las periferias de las ciudades, los cuales competían con las áreas comerciales de los centros urbanos (Espinosa, 2003).

Los centros urbanos se han convertido en áreas de atracción de ciudadanos por la diversidad funcional, y es donde se localizan los comercios, los equipamientos de alta jerarquía, los centros financieros, los centros de alto valor histórico y patrimonial, los enclaves simbólicos y los escaparates de las relaciones de poder entre la sociedad y las élites gobernantes, entre otros (Somoza-Medina 
y López-González, 2017; Venturini, 1992). Estos espacios de comercio y consumo actúan como lugares clave para la producción de identidad (Abelson, 1989; Benson, 1986; Cornide, 2020).

La localización espacial de los centros urbanos se caracteriza por la centralidad, porque es donde el espacio público es más diverso e intensivo, por tener más puntos de acceso y estar próximo a otros usos complementarios (Bros et al., 1995), o con un mínimo desplazamiento, aun estando sujeto a cambios en la distribución de la población mientras crece la ciudad (Northam, 1975).

$\mathrm{Al}$ tener en cuenta las actividades comerciales y de servicio y el flujo de personas es cuando la localización geográfica de los centros adquiere sentido. En primer lugar, los centros urbanos se configuran como espacios polivalentes donde lo que ocurre determina la vida de los habitantes de la ciudad y en todas sus esferas, según Paris (2013). A pesar de la descentralización de equipamientos hacia la periferia, se mantienen las funciones centrales políticas y administrativas, económicas, comerciales y sociales. Rangel (2009) incluye la presencia de elementos simbólicos de referencia de la ciudad como un indicador de calidad en espacios públicos. El simbolismo del espacio urbano atrae a peatones con finalidades distintas, que a su vez generan movimientos e intensifican el uso variado del espacio público. La función residencial sería ahora una de las prioridades de gobiernos locales para recuperar el vigor en el marco de la diversificación funcional (Zárate, 2006).

En segundo lugar, los centros urbanos coinciden espacialmente con las áreas de mayor densidad de patrimonio histórico y cultural. En este contexto, se ponen en valor estos bienes, la necesidad de políticas de conservación y nuevos indicadores de análisis, entre otros (Guzmán et al., 2017; García, 2014), para una gestión y un desarrollo local sostenible en sus tres dimensiones: económica, social y ambiental. Los estudios con recuento de peatones establecen una relación directa positiva con los edificios históricos (Park et al., 2018). De igual forma, el hecho de preservar o añadir nuevos usos cotidianos a este tipo de equipamientos supone la revalorización de conjunto del barrio o de una calle. Un estudio sobre el comercio local en el centro histórico de Reus, en Cataluña (Castellà et al., 2018), sostiene la relación inherente entre el patrimonio catalogado y el comercio local como un argumento de peso para destacar la singularidad de un determinado centro histórico urbano.

Al proceso de degradación y abandono de muchas áreas centrales, le han seguido proyectos de rehabilitación urbana. Segado y Espinosa (2015) recogen intervenciones de éxito que aumentaron la frecuentación y la diversidad social y cultural a partir de la renovación de vivienda en edificios antiguos y de nueva construcción, actuaciones combinadas con equipamientos para servicios públicos y actividad comercial (comercio, restauración, servicios, oficinas, almacenamiento, artesanía y pequeño-industrial). Es más, se trata de la concepción del barrio centro histórico como monumento unitario a preservar. La rehabilitación urbana y la revitalización comercial son dos procesos que deben darse en paralelo (Elizagarate y Zorrilla, 2004). La excesiva intervención que ha dado lugar a la tematización del centro urbano, la gentrificación y la 
desertización de los usos tradicionales termina generando centros infrautilizados o muy concurridos puntualmente, monofuncionales o museizados, donde la actividad comercial se transforma en formato sucursalista o franquiciado (Rebollo, 2003).

En definitiva, la centralidad, la accesibilidad y la conectividad que caracterizan estas áreas incitan a la frecuentación peatonal acorde con la diversidad funcional que albergan, la calidad, la conservación y la mixtura de usos también en los equipamientos históricos.

\subsection{El peatón, la calle y la actividad comercial}

El urbanismo moderno tiende a incluir en sus proyectos el uso que los peatones hacen del espacio público y la interacción social, que puede verse favorecida o coartada por la calidad del diseño y las condiciones de las calles, para garantizar la afluencia de peatones.

Este fenómeno fue estudiado por primera vez por Jacobs (1961), que consideraba la «muerte» de la calle, entendida como el espacio de la interacción social, a causa del predominio del automóvil. Jacobs estableció las características para que la calle acogiera distintos usos y usuarios, que sintetizaba en factores económicos (localización de distintas actividades y rentas), morfológicos (edificios y calles) y de densidades residenciales. La combinación correcta de estas características conlleva a un uso de la calle. Se han aplicado los criterios de Jacobs para identificar, en otros contextos espaciales y temporales, la vitalidad de las calles, por ejemplo en ciudades mediterráneas (Delclòs y Miralles, 2018).

A partir de dichas bases, aparece numerosa literatura científica que profundiza en algunos de los criterios necesarios para la existencia de una calle «viva». Un elemento es la existencia de un espacio físico que favorezca la presencia de personas para hacer múltiples actividades (Gehl y Svarre, 2013). La calle debe ser accesible no solo en automóvil privado, sino especialmente con medios de transporte públicos (Di Ciommo y Lucas, 2014), y garantizar el confort y el uso atractivo peatonal (Pozueta et al., 2009; Talavera-García et al., 2014). En este sentido, la pacificación del tráfico es esencial para garantizar la seguridad vial (Sanz, 1996). La conjunción de factores posibilita el atractivo para que se dé lo que Gehl (2006) denomina actividades sociales o la posibilidad de «serendipia» (Lavadinho, 2014).

La presencia de actividad en los locales de planta baja es también uno de los elementos que señalaba Jacobs (1961) como punto de atracción de peatones. Ya en 1989, Bourjaillat y Rabolloud (1989) se referían a la complementariedad de la peatonalización y las áreas de pequeño comercio. Estudios posteriores (Simó et al., 2018) resaltan que las áreas comerciales en calles peatonales resistieron mejor la crisis económica que las actividades ubicadas en otros espacios. En ese mismo contexto, el trabajo de Hahm, Yoon y Choi (2019) determina que las condiciones de calidad del espacio público en las calles comerciales están relacionadas directa y positivamente con las actividades de caminar y comprar. 


\section{Metodología y datos}

\subsection{Datos y fuentes}

Con el objetivo de analizar la afluencia de peatones en los centros comerciales urbanos de siete ciudades medias catalanas, se han seleccionado 19 variables que se clasifican en cuatro indicadores: 1) la movilidad peatonal que hace referencia a la movilidad diaria de peatones (IMD) (una variable); 2) la actividad económica que clasifica los sectores comerciales, los servicios y los locales abiertos y cerrados (nueve variables); 3) la morfología donde se definen las características del espacio público, desde la orientación de las calles hasta su amplitud o el tipo de tránsito (cuatro variables), y 4 ) incluye las variables que definen la distancia de los centros urbanos con los servicios de transporte público (bus, tren) y equipamientos de la ciudad (cinco variables) (ver tabla 1).

La Fundació Comerç Ciutadà (FCC) aporta información, para el periodo 2017-2019, sobre la monitorización de las calles, su estructura y sus atributos, y el censo de establecimientos comerciales ubicados a pie de calle. El resto de las variables se ha obtenido de los datos abiertos de cada una de las ciudades.

\subsection{Análisis clúster para la clasificación de los centros urbanos}

El análisis clúster (CL) consiste en agrupar un conjunto de observaciones en un número de clústeres basándose en la distancia mínima. Se utiliza en estudios sobre actividad comercial (Elizagarte et al., 2005), áreas urbanas (Nosoohi y Zeinal-Hamadani, 2011), para agrupar ciudades pequeñas y medianas según la intensidad de urbanización (Stoica et al., 2020) y para la gestión del territorio (Nunes, 2002; Serra et al., 2014). Y, más recientemente, el CL agrupa, a partir de las variables que definen los cuatro indicadores, las calles con una dinámica similar, a pesar de las diferencias funcionales y territoriales que las separan (Johnson y Wichern, 2007; Myint, 2008). Para el cálculo de la distancia entre los grupos, se ha utilizado el método de Ward (W) porque, a diferencia de otros métodos de agrupación, no emplea la matriz de distancia, sino que se calcula a partir de la distancia euclidiana al cuadrado entre cada elemento y la media del grupo al que pertenece.

$$
\mathrm{W}=\sum_{\mathrm{g}=1}^{\mathrm{G}} \sum_{\mathrm{i}=1}^{\mathrm{n}_{\mathrm{g}}}\left(\mathrm{x}_{\mathrm{ig}}-\overline{\mathrm{x}}_{\mathrm{g}}\right)\left(\mathrm{x}_{\mathrm{ig}}-\overline{\mathrm{x}}_{\mathrm{g}}\right)^{\prime}
$$

Donde:

$\overline{\mathrm{x}}_{\mathrm{g}}$ es la mediana del grupo $\mathrm{g}$

$G$ es cada grupo.

La distancia euclidiana es utilizada por su condición para clasificar casos de distinta naturaleza, tratados como unidades de análisis diferenciado e inde- 
Tabla 1. Descripción de las variables y fuentes de datos

\begin{tabular}{|c|c|c|c|}
\hline Indicadores & Variable & Descripción & Fuente \\
\hline Movilidad peatonal & IMD & $\begin{array}{l}\text { Intensidad media diaria: suma de peatones monitorizados } \\
\text { en } 24 \text { horas }\end{array}$ & FCC \\
\hline \multirow[t]{9}{*}{ Dinámica comercial } & LCT & Locales comerciales cerrados & \multirow{9}{*}{$\begin{array}{l}\text { Censo de locales } \\
\text { en planta baja, por } \\
\text { categorías de actividad. } \\
\text { FCC }\end{array}$} \\
\hline & $\mathrm{LCO}$ & Locales comerciales abiertos & \\
\hline & QA & Cuotidiano alimentario & \\
\hline & QNA & Cuotidiano no alimentario & \\
\hline & EQP & Equipamiento moda personas & \\
\hline & EQLL & Equipamiento del hogar & \\
\hline & OLLIC & 0cio, entretenimiento y cultura & \\
\hline & RTA & Restauración y hostelería y comida para llevar & \\
\hline & SERV & Servicios a las personas, a la salud y profesionales y técnicos & \\
\hline \multirow[t]{5}{*}{ Morfología } & ORIENT & $\begin{array}{l}\text { Grados de orientación natural de la calle respecto del norte } \\
1^{\circ} \text { a } 360^{\circ} \text {. }\end{array}$ & Elaboración propia \\
\hline & \multirow[t]{2}{*}{ ESTR } & Estructura calle según estandarización 1 a 7: & \multirow[b]{2}{*}{ FCC } \\
\hline & & $\begin{array}{l}1 \text { = peatonal hasta } 4 \mathrm{~m} \text { ancho. } 2=\text { peatonal de } 4 \text { a } 8 \mathrm{~m} \text { ancho. } \\
3=\text { peatonal de } 4 \text { a } 8 \mathrm{~m} \text { de ancho con mobiliario. } 4=\text { motori- } \\
\text { zada y aceras de } 4 \text { a } 8 \mathrm{~m} \text { ancho. } 5=\text { peatonal y ancho }>8 \mathrm{~m} \\
\text { de doble } 0 \text { triple sección. } 6=\text { motorizada y ancho }>8 \mathrm{~m} \text { con } \\
\text { doble } 0 \text { triple sección. } 7=\text { peatonal y ancho }>8 \mathrm{~m} \text { de doble } 0 \\
\text { triple sección con mobiliario. }\end{array}$ & \\
\hline & ATRIBUTS & $\begin{array}{l}\text { Cualidades del espacio público según estandarización } 1 \text { a 3: } \\
1 \text { = calle de tránsito motorizado de acera estrecha o inexistente. } \\
2 \text { = calle de tránsito motorizado de acera ancha, árboles y } \\
\text { mobiliario urbano. } 3 \text { = peatonal plataforma ancha, árboles y } \\
\text { mobiliario. }\end{array}$ & FCC \\
\hline & TRANS & $\begin{array}{l}\text { Tránsito dominante estandarización } 1 \text { a 2: } 1 \text { = peatonal. } 2 \\
=\text { libre motorizado }\end{array}$ & Elaboración propia \\
\hline \multirow[t]{5}{*}{ Funcionalidad } & EQUIP & Equipamientos públicos y privados & Datos abiertos ayto. \\
\hline & DPA & Distancia mínima a estacionamiento, aéreo o subterráneo & \multirow{4}{*}{$\begin{array}{l}\text { Elaboración propia a tra- } \\
\text { vés servidor aplicativo de } \\
\text { mapas web }\end{array}$} \\
\hline & MTF & $\begin{array}{l}\text { Distancia mínima a estación de movilidad interurbana } \\
\text { (ferrocarril o autobuses) }\end{array}$ & \\
\hline & MTB & Distancia mínima a parada bus urbano & \\
\hline & DMM & Distancia mínima a mercado municipal & \\
\hline
\end{tabular}

Fuente: elaboración propia.

pendiente espacialmente del resto de casos de estudio y reagrupados en conglomerados sólidos en rasgos diferenciales y con una composición interior lo más homogénea posible.

Para identificar el número de clústeres previamente, se ha elaborado un dendrograma a partir de la distancia euclidiana. Del resultado se han identificado seis grupos. Una vez se han especificado las variables y los grupos de calles similares de las siete ciudades medias, se han validado los resultados a partir de técnicas cualitativas de observación y fotodocumentación de las áreas de estudio. 


\section{Resultados: clústeres de las calles de las ciudades medianas}

Los resultados del análisis clúster muestran, a partir de la agrupación de las 19 variables, seis clústeres (tabla 2) que reagrupan las 82 calles de estudio según las características que definen su movilidad peatonal, la dinámica comercial, la morfología urbana y la funcionalidad. La definición de cada clúster se ha realizado teniendo en cuenta los valores medios resultantes del análisis y las observaciones del trabajo de campo.

\section{Clister 1: corredores con actividad comercial}

El primer clúster está formado por 15 de las 82 calles, representa el $20 \%$ del total de metros analizados en el estudio (3.220 m de longitud de calle) y se halla en todos los centros urbanos a excepción de Olot.

Los principales indicadores que identifican estas calles son la movilidad peatonal y la accesibilidad a los servicios (figura 2). La dinámica comercial muestra una diversificación de la actividad. Si bien los comercios de equipamiento de moda de personas y los locales que ofrecen servicios seguidos de la restauración ocupan buena parte de las plantas bajas, la concentración según sectores es baja, y en estas calles es fácil hallar una perfumería o una zapatería compartiendo fachada con una ferretería, una granja, una farmacia y una consultoría. Predominan los locales abiertos.

Tabla 2. Cuadro resumen de los valores centrales de los seis clústeres

\begin{tabular}{llrrrrrr}
\hline Indicadores & Variables & \multicolumn{1}{c}{ CL1 } & \multicolumn{1}{c}{ CL2 } & \multicolumn{1}{c}{ CL3 } & \multicolumn{1}{c}{ CL4 } & \multicolumn{1}{c}{ CL5 } & \multicolumn{1}{c}{ CL6 } \\
\hline Mov. Peatones & IMD & $9.495,73$ & 7145 & $5.223,56$ & 3744,41 & 1782,17 & $\mathbf{1 . 6 4 3 9 , 8 9}$ \\
\hline Dinámica & LCT & 4,13 & 2,64 & 3,44 & 4 & 3,67 & 4,22 \\
comercial & LCO & 36,93 & 32,09 & 23,06 & 20,71 & 17,58 & 61,11 \\
& QA & 3,6 & 4,09 & 2,83 & 1,71 & 1,75 & 4,78 \\
& QNA & 2,73 & 2,36 & 1,33 & 1,65 & 0,92 & 5,33 \\
& EQP & 11,8 & 12,36 & 6,61 & 5,59 & 5,33 & $\mathbf{2 2 , 8 9}$ \\
& EQLL & 1,67 & 2,36 & 1,72 & 1,71 & 0,75 & 5,33 \\
& OLLiC & 2,2 & 1,09 & 0,72 & 1,12 & 1 & 2,56 \\
& RTA & 5,73 & 2,82 & 3,28 & 1,88 & 2,08 & 6,67 \\
Morfología & SERV & 7,2 & 6,36 & 5,67 & 6,53 & 5,25 & $\mathbf{1 2 , 5 6}$ \\
\hline & ORIEN & 189,53 & 264,18 & 135,28 & 225,77 & 251,92 & 250,44 \\
& ESTR & 3,47 & 2,73 & 3,44 & 2,65 & 3,33 & 5,56 \\
& ATRIB & 2,13 & 1,55 & 2,11 & 1,77 & 1,67 & 2,33 \\
& TRANS & 1,4 & 1,46 & 1,39 & 1,53 & 1,5 & 1,56 \\
\hline Funcionalidad & EQUIP & 1,07 & 1,46 & 1,11 & 1,06 & 0,67 & 1,33 \\
& DPA & 261,33 & 338,27 & 211,22 & 287,24 & 243,33 & 258,11 \\
& MTF & 663,47 & 659,09 & 578,33 & 782,35 & 563,33 & 552,22 \\
& MTB & $\mathbf{1 8 6 , 4 7}$ & 184,55 & 195,61 & 273,88 & 202,5 & 186 \\
& DMM & $\mathbf{5 3 0 , 6 7}$ & 425,36 & 371,28 & 391 & 360,83 & 373,89 \\
\hline
\end{tabular}

Fuente: elaboración propia a partir de la síntesis de cuadros resultantes de valores para cada clúster. 
Figura 2. Gráfica de la dinámica general de los casos incluidos en el clúster 1

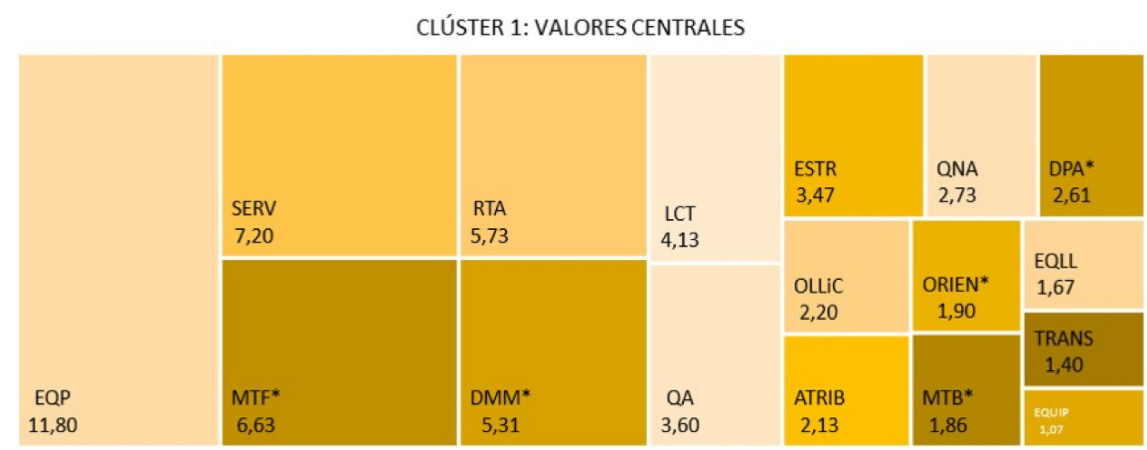

* La gráfica excluye los valores máximos que hacen referencia a la movilidad de peatones y se han estandarizado los valores indicadores de funcionalidad.

Fuente: elaboración propia.

La morfología de las calles es de estructura ancha, de recorrido medio-largo, con esquinas frecuentes. Por su orientación natural, principalmente son calles sombrías, pero poco condicionadas por su tipología edificatoria debido a su anchura — de más de 10 metros. El conjunto reúne casos peatonales —más estrechos- y otros pacificados que permiten el tránsito rodado. Aunque el espacio público dispone de elementos de calidad para facilitar el paso, no es el mejor equipado en mobiliario. Este último factor limita la posibilidad de largas estancias o de actividades de recreo al uso privado de las terrazas exteriores propias de la actividad de la restauración, en algunos casos.

Finalmente, la funcionalidad presenta importantes corredores de peatones y enlaces clave para el transporte intermodal, como la calle Tres Creus, en Sabadell. Se trata de calles bien comunicadas, con varias líneas de bus o de transporte ferroviario, o por carretera interurbano, además de su proximidad a la oferta de estacionamiento, lo que facilita la entrada al centro con el vehículo particular. En síntesis, garantizan el flujo de visitantes urbanos e interurbanos. La combinación de los usos residencial — de edificación media y alta-, laboral y comercial y la presencia de importantes equipamientos públicos - bibliotecas, iglesias, ayuntamientos, museos - en su trayectoria coincide con el hecho de que alrededor de 10.000 personas transiten estos espacios a diario.

\section{Clúster 2: calles comerciales tradicionales}

El segundo clúster define 11 casos, un $12 \%$ del total $(1.917 \mathrm{~m}$ de calles analizadas) (figura 3). Hay calles en este clúster en todos los centros analizados, excepto en el de Sabadell.

La dinámica comercial de este clúster tiene como rasgo común la concentración de comercios de proximidad en establecimientos históricos. La espe- 
Figura 3. Gráfica de la dinámica general de los casos incluidos en el clúster 2

CLÚSTER 2: VALORES CENTRALES

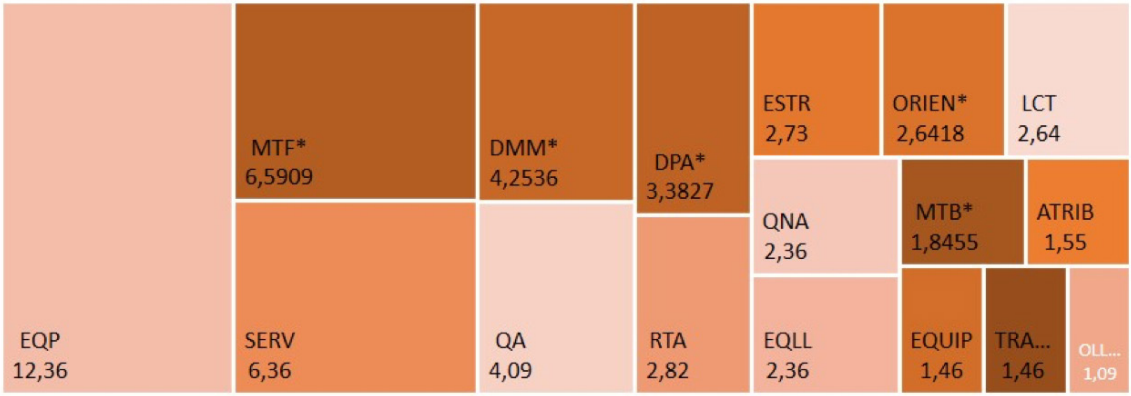

* La gráfica excluye los valores máximos que hacen referencia a la movilidad de peatones y se han estandarizado los valores indicadores de funcionalidad.

Fuente: elaboración propia.

cialización comercial no se da por categorías, aunque presenta un valor central muy alto de comercios de producto cotidiano alimentario (QA-4.09), en estos casos, de producto artesanal y de gran valor añadido por la proximidad de la cadena de elaboración y la calidad del producto final, característica de la calle Argenters en Vic, por ejemplo. Pero esta última característica también se extiende al resto de categorías y sectores, en particular, al equipamiento de moda de personas y al equipamiento del hogar, o incluso a la hostelería. Asimismo, el valor bajo de esta última actividad —la restauración y hostelería (RTA-2.82) - puede explicarse por la morfología, el tipo de edificación y la falta de espacio exterior para la implementación de terrazas, sin ir más lejos.

La morfología presenta calles sombrías, más por su trama estrecha - muy inferior a los $8 \mathrm{~m}-$ e irregular que por su orientación natural o por la tipología de edificación, relativamente baja, de planta estrecha $+2 o+3$ pisos. Se trata de calles de plataforma única, peatonales $y$, en general, limitadas a las acciones de pasar, con escaso mobiliario y con menos opciones para la ocupación del suelo por parte de las terrazas de cafeterías y restaurantes.

La funcionalidad tiende a la distribución espacial transversal, formando pasillos conectores entre el centro urbano con el núcleo histórico patrimonial e incluyendo equipamientos de primer orden administrativo y cultural. El uso intensivo del espacio público se refleja en un valor central que se sitúa en torno a los 7.000 transeúntes diarios.

Se trata de calles de recorrido corto-medio, como en el clúster 1, con esquinas frecuentes que favorecen el flujo peatonal. Aunque la distancia a los distintos servicios de movilidad no es de las más bajas de la muestra, sí permite la accesibilidad a los diversos modos de transporte, incluidas las estaciones de líneas interurbanas, ferroviarias o por carretera, a más de 600 metros (MTF-6.59) de distancia y con estacionamientos a más de 300 metros (DPA-3.38). 


\section{Clústeres 3 y 4: de las puertas comerciales peatonales a la baja densidad comercial}

En el caso de los clústeres 3 y 4 , tienen características parejas. En total, definen a 18 y 21 calles respectivamente, e incluyen los bloques más numerosos del estudio; por un lado, el $22 \%$ de la muestra ( $3.646 \mathrm{~m}$ de calles) y, por el otro, el 16\% (2.684 m) (figuras 4 y 5). Esta tipología se encuentra en las siete áreas de estudio, a excepción del clúster 4, que no tiene representación en Vic.

Las características son las siguientes. La dinámica comercial muestra una diversidad de usos comerciales, donde los servicios y los equipamientos de moda de personas tienden diluirse entre el resto de categorías y sectores. Este rasgo es más evidente en el clúster 4, donde, por ejemplo, la restauración y la hostelería quedan por delante de los comercios de ocio y cultura y los equipamientos. Otro valor importante es el elevado número de locales comerciales cerrados (LCT-3.44 y 4.00) y la discontinuidad de fachada comercial ocupada por otros equipamientos, como en la calle de la Rasa en Terrassa.

Figuras 4 y 5 . Gráfica de la dinámica general de los casos incluidos en los clústeres 3 y 4

CLÚSTER 3: VALORES CENTRALES
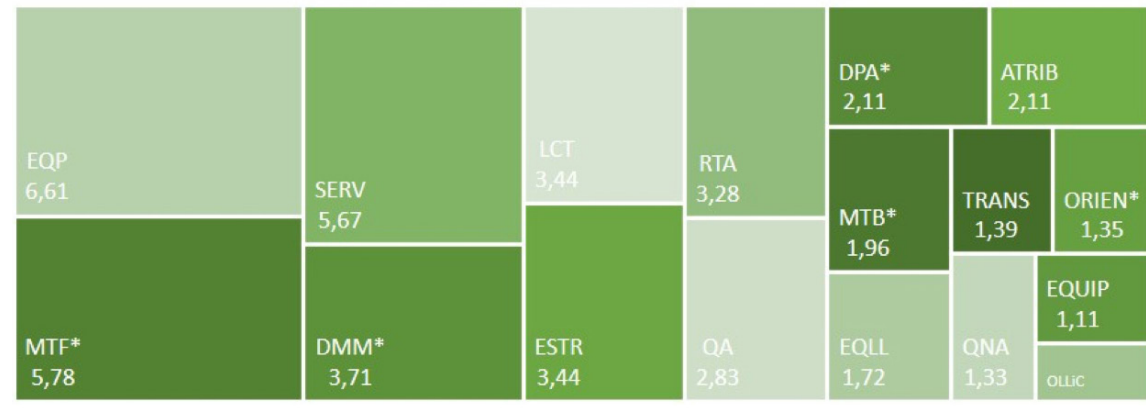

CLÚSTER 4: VALORES CENTRALES
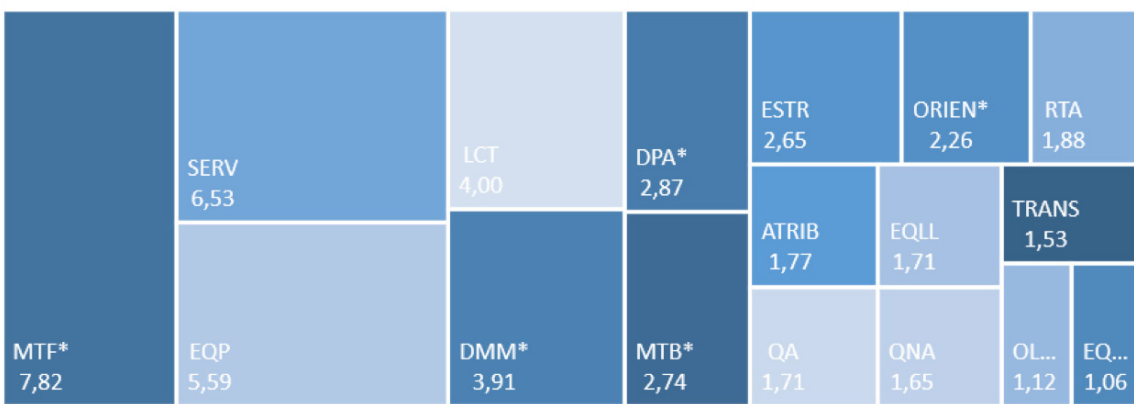

* La gráfica excluye los valores máximos que hacen referencia a la movilidad de peatones y se han estandarizado los valores indicadores de funcionalidad.

Fuente: elaboración propia. 
Las variables definen la morfología de las calles con una estructura entre los 8 y los 10 metros de ancho mínimo entre fachadas. La calidad del espacio público del clúster 3 y el 4 tiene una la plataforma única: la vegetación ornamental y puntos de mobiliario concentrados espacialmente para el contacto social puntual (entrada-salida de centros académicos y culturales), que también varía entre ambos clústeres. En este sentido, se contempla desde la movilidad peatonal y pacificada de la mayoría de los casos hasta una menos restrictiva circulación libre motorizada, donde las aceras estrechas dificultan el paso, individual y en grupo, en menor proporción. El resultado centroide de la variable orientación las sitúa en ángulos opuestos y de igual modo más sombrías que soleadas.

La funcionalidad indica que estos casos tienden a localizarse fuera del núcleo central y conecta la periferia motorizada con el acceso peatonal al centro. Los equipamientos y servicios culturales —en su caso, escuelas, academias, entidades culturales, museos y casinos - se combinan con el uso residencial de baja densidad edificatoria, lo que deja entrever un paisaje de transición de usos, como en la calle Príncep de Viana de Granollers. Estas dinámicas coinciden con la reducción del uso del espacio público, que pasa de mantenerse transitado con 5.200 peatones diarios en el clúster 3 a 3.700 en el clúster 4. Tienen una accesibilidad de recorrido corto-medio, con conexión frecuente a las vías adyacentes. En general, están mejor comunicadas con los servicios a la movilidad local (bus y aparcamiento) que a la interurbana (MTF-782). Asimismo, los casos del clúster 3 son de los más próximos al mercado municipal, por detrás del clúster 5, mientras que las calles del conglomerado 4 tienden a un mayor distanciamiento respecto a este (DMM-371 y 391).

\section{Clúster 5: calles interiores residenciales}

El quinto clúster incluye 12 calles de los siete centros urbanos, que representan el 13\% del total de metros de calle $(2.150 \mathrm{~m}$ ) (figura 6). No es posible identificar estas categorías entre las calles de análisis en los centros de Mataró y de Terrassa.

La dinámica comercial es escasa y presenta una gran discontinuidad de fachada comercial porque la función residencial es predominante. En consecuencia, los locales en activo se encuentran dispersos, pero, a diferencia del clúster anterior, tienden a concentrar una sola categoría y sector comercial. Así, dentro de este conjunto encontramos calles de restaurantes o calles de equipamientos de moda o calles de servicios profesionales y técnicos o calles de comercio tradicional. En cualquier caso, se trata de negocios familiares. Por otro lado, sus casos son los más próximos al mercado municipal (DMM-360,73) de todo el estudio y la competitividad por categorías es la menor de la muestra. La dinámica comercial y el tipo de establecimiento están vinculados al tipo de edificación (estrecha y baja) y a la todavía pendiente necesidad de rehabilitación estructural que muestran algunas construcciones. 
Figura 6. Gráfica de la dinámica general de los casos incluidos en el clúster 5

CLÚSTER 5: VALORES CENTRALES

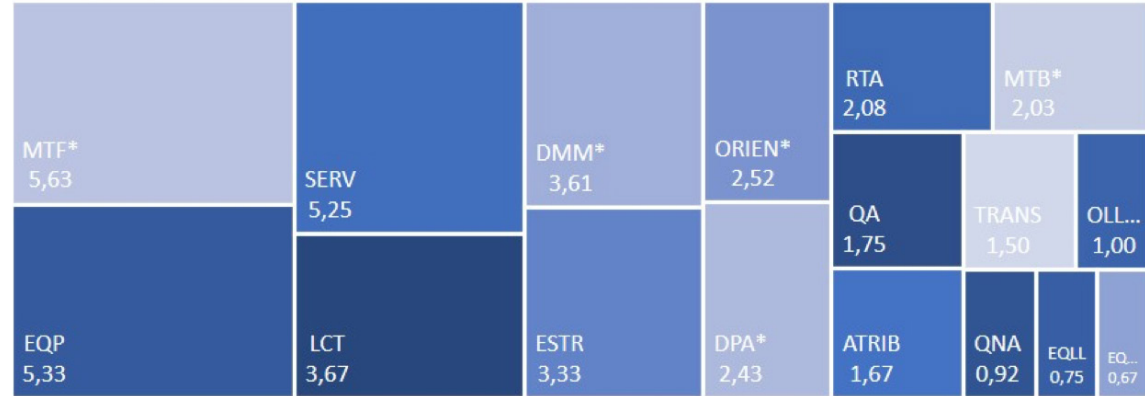

* La gráfica excluye los valores máximos que hacen referencia a la movilidad de peatones y se han estandarizado los valores indicadores de funcionalidad.

Fuente: elaboración propia.

Las características morfológicas de las calles son las mismas que las del clúster 2, ya que se trata de calles relativamente estrechas e irregulares y peatonales. Se encuentran concentradas en el centro histórico y, salvo una excepción, no disponen de equipamientos públicos de primer orden, o no se encuentran en su trayectoria directa.

La funcionalidad de las calles del clúster 5 son, en su mayoría, de paso alternativo, donde domina el uso residencial de baja densidad. En algunas se alterna la conservación - de fachadas y detalles históricos en buen estado, pavimento, alumbrado o señalización - con el deterioro y el abandono de edificación privada. La limitación de usos al residencial se refleja en el valor centroide del tránsito de peatones, entorno a los 2.000 diarios, como muestra la calle Sastres en Olot.

Conectividad y accesibilidad. Se mantienen los buenos niveles de comunicación de las calles del clúster 2 , incluso con más reducción de la distancia a una estación de autobuses de movilidad interurbana (MTF-5.63) o de la red ferroviaria. Es ligeramente superior hasta las líneas urbanas de bus, entorno a los 200 metros, y con un buen acceso para el aparcamiento del vehiculo privado entorno a la misma cifra.

\section{Clister 6: ejes centrales comerciales}

El sexto y último clúster es el más pequeño y agrupa 9 casos, que suponen el $17 \%$ del total de metros $(2.776 \mathrm{~m})$. Este tipo de calles resultó en cinco centros y dejó fuera Figueres y Olot.

La dinámica comercial presenta un modelo comercial intensivo, donde la mixticidad de usos comerciales caracteriza las plantas bajas de estas calles. Ahora bien, al tratarse de calles largas, es notable la existencia de tramos especializados o con disposición para que distintos tipos de establecimientos pre- 
Figura 7. Gráfica de la dinámica general de los casos incluidos en el clúster 6 CLÚSTER 6: VALORES CENTRALES

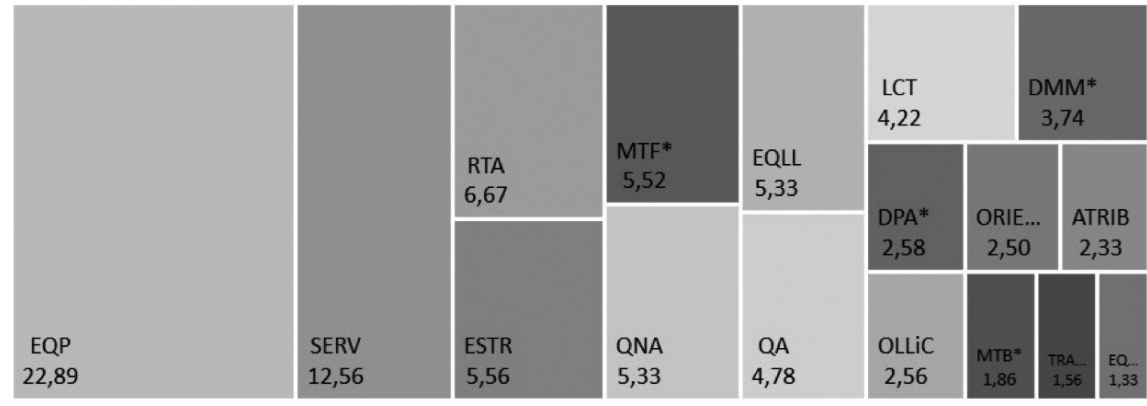

* La gráfica excluye los valores máximos que hacen referencia a la movilidad de peatones y se han estandarizado los valores indicadores de funcionalidad.

Fuente: elaboración propia.

dominantes, como los equipamientos de moda de personas (ropa, ópticas, perfumerías zapaterías y complementos), compartan fachada, y otros, como la restauración con terraza exterior - concentrados en una acera o dispersos en los extremos- coexistan con locales de todo tipo de servicios — a las personas, a la salud o profesionales y técnicos. Otro rasgo importante de la dinámica comercial de este clúster es la concentración de firmas y cadenas nacionales y multinacionales. El modelo sucursalista y franquiciado se extiende en el sexto clúster a todos los sectores, no solo al de moda y complementos, sino también a los de la restauración, los servicios — como peluquerías, librerías - e incluso al del producto cotidiano no alimentario patente en grandes locales de telefonía. En este sentido, categorías como los comercios de equipamiento para el hogar, que incluyen mobiliario y decoración, se encuentran muy por encima de la media, incluso comparten peso con otras tipologías de comercios, como el cotidiano alimentario y el no alimentario.

La morfología urbana se caracteriza per calles largas y las más anchas entre fachadas - más de $10 \mathrm{~m}$-, por lo que su orientación natural no impide ni las aceras soleadas ni las sombrías. Además, coinciden con vías longitudinales y transversales, también formando ejes que permiten cruzar el centro urbano de un extremo al otro. La tipología edificatoria, es relativamente alta - planta +5 pisos-, lo que permite el uso comercial a pie de calle y garantiza una mayor continuidad de escaparates. El diseño de los casos de este grupo es diverso, de plataforma única o pacificado, pero con amplias aceras, bien dotadas de árboles o vegetación ornamental y bien equipadas de mobiliario, orientado tanto al paso como a las largas estancias y al desarrollo de actividades de socialización o contemplación.

La funcionalidad indica que se trata de arterias del centro urbano, largos ejes donde se combinan y concentran múltiples y diferentes usos primarios -residencial (medio-alto) y administrativo y cultural o de abastecimiento de 
Figura 8. Estructura de los centros urbanos y clústeres resultantes
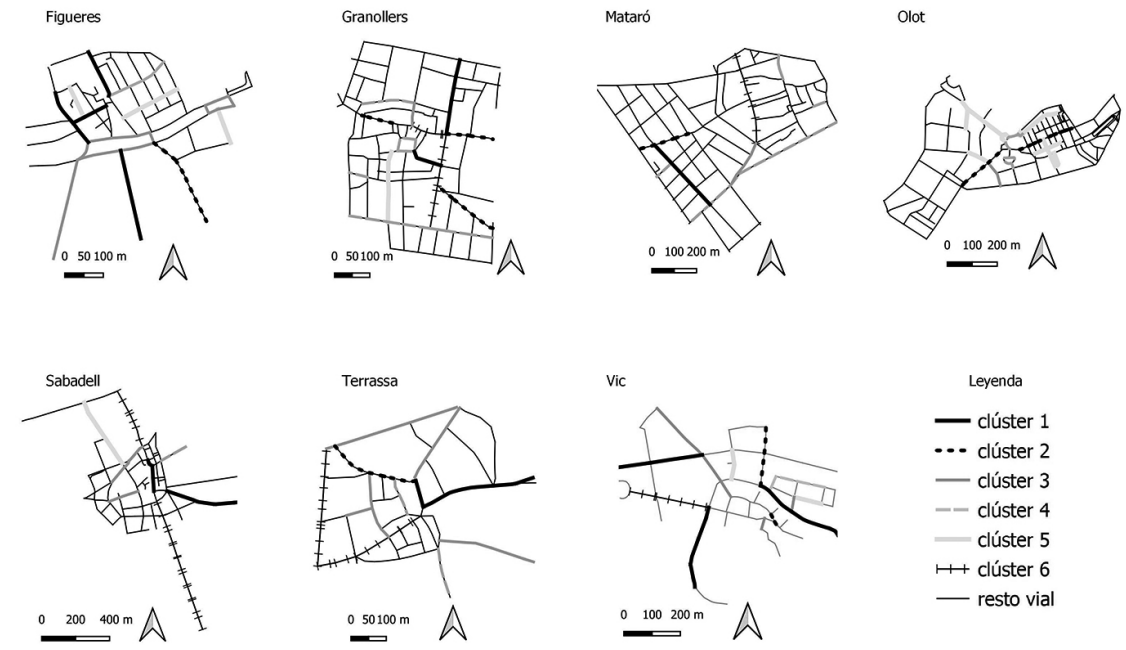

Fuente: elaboración propia con las bases cartográficas del Instituto Cartográfico y Geológico de Cataluña (ICGC) y el sistema de información geográfica QGIS.

primer orden, como los mercados municipales — con usos secundarios — todo tipo de comercio y servicios - para atender las demandas de los primeros. La mixtura de usos de los casos coincide con el valor centroide más alto de la variable movilidad peatonal de todo el estudio. Son las calles principales donde a diario transitan más de 16.000 peatones, entre otras, la Riera en Mataró. La accesibilidad indica que son casos de recorrido largo, pero que cuentan con intersecciones frecuentes que facilitan la función conectora con el resto del centro urbano y exterior de forma rápida. El análisis indica que son las calles mejor comunicadas del estudio con los principales servicios de movilidad y registran los valores más bajos (líneas de autobús a una distancia de $186 \mathrm{~m}$ y transporte interurbano ferroviario o por carretera a $552 \mathrm{~m}$ ). Del mismo modo, el clúster 6 es el segundo más accesible del estudio a través de la oferta de estacionamiento, por detrás del tercer clúster.

A continuación, la figura 8 ofrece el detalle de los seis clústeres para cada uno de los centros urbanos.

\section{Discusión: la frecuentación del espacio público y su organización comercial}

El flujo de peatones, la morfología urbana y la organización comercial son factores complementarios que ayudan a explicar la localización espacial de la actividad comercial de los centros urbanos. Es posible identificar dentro de cada área de estudio clústeres de calles que responden a funcionalidades, dinámicas y morfologías distintas. 
En todos los clústeres, la movilidad de peatones es el indicador que presenta los valores más altos, y se da este hecho porque en todos los casos es fácil el acceso a los espacios comerciales en transporte público (local o interurbano) y a ofertas de estacionamiento para el vehículo privado (Di Ciommo y Lucas, 2014; Broset al., 1995). Además, para la localización óptima de empresas de servicios, es importante la proximidad de redes de transporte de personas, tanto públicas como privadas, para la interacción socioeconómica (Entremont, 1997). Así los clústeres de baja densidad comercial son aquellos donde hay un menor flujo de peatones.

En la dinámica comercial inciden las características del espacio público y la morfología urbana. Como se observa en los ejes comerciales (clúster 6), son calles con una alta frecuentación de peatones, donde también se encuentran los monumentos históricos, los espacios de aglomeración comercial para la distribución de bienes y servicios especializados y los principales equipamientos y servicios. A pesar de haber sido intervenida la morfología urbana con la peatonalización o cambios en la ornamentación de las fachadas, se mantiene la actividad comercial, o incluso se ha revitalizado (Segado y Espinosa, 2015). Así, la diversidad de usos, los cambios en la morfología y la alta conectividad son tres elementos importantes para la implementación de la actividad económica; en particular, surtida de distintos tipos de sector comercial y, en general, especializada en el negocio sucursal de cadenas y firmas internacionales a las que hacía referencia Rebollo (2003).

Las calles altamente frecuentadas con actividad comercial (clúster 2) y calles interiores donde se encuentran espacios residenciales (clúster 5) tienden a configurarse como circuitos de nuevos espacios de actividad económica en consonancia con los nuevos valores de la sociedad posindustrial (Entremont, 1997). Se trata de espacios en los que cobra interés el recurso humano (el trabajo y la creatividad de las personas a las que apunta el autor), visible en los comercios de producto de proximidad de todo tipo, no solo en el alimentario, sino también en el de la fabricación artesana que tiene en cuenta los valores medioambientales que captan la atención de la sociedad actual y que ganan presencia en las calles. El valor añadido de este conjunto es más evidente en la conservación de sus elementos históricos y patrimonio arquitectónico, donde la intervención para su conservación o rehabilitación ha sido más respetuosa, hasta el punto de dejar intacta la trama y ofrecer la alternativa del paseo, así como la posibilidad de "serendipia» a la que hace referencia el autor Lavadinho (2014).

Las calles con menor actividad comercial y con menor afluencia de peatones son las que presentan una dinámica residencial más elevada. En sí mismas, las variables con las que se ha trabajado no pueden explicar dicho fenómeno. Sin embargo, la observación de campo, caracterizada por la espontaneidad y la diversidad de franjas horarias en las que se produjo, evidencia que las calles vivas de este estudio y las de prácticamente el resto de clústeres muestran una connotación bien distinta a la de Jacobs (1961), a falta de indicios de cotidianidad, de identidad, de signos de vecindad que solo puede aportar un uso residencial consolidado que - sin determinar estadísticamente ni comprobar su uso real para cada uno de los casos - no se reconoce en el ambiente. 


\section{Consideraciones finales}

La investigación de carácter exploratorio se plantea inicialmente el análisis en 82 calles de siete centros urbanos. Los resultados muestran seis clústeres que presentan patrones de distribución comercial sectorial, funcional y de atributos del espacio público similares que atraen una determinada afluencia diaria de peatones. A partir de los seis clústeres, se concluye que en los siete centros urbanos pueden identificarse tres tipos de organización comercial, estructura física y su relación con la intensidad del flujo de peatones.

El primero se caracteriza por el uso comercial intensivo y diverso a pie de calle y especializado en firmas multinacionales. Coincide con las áreas peatonales, que históricamente son centros de aglomeración y distribución de bienes y servicios, y se trata de los espacios con mayor accesibilidad y comunicación con los distintos servicios de la movilidad (transporte público de ámbito local e interurbano o privado) y de los más frecuentados.

El segundo se identifica con un legado histórico y una elevada densidad comercial, centrada en los modelos de negocio familiar, que tienden a erigirse como nuevos espacios para la creatividad donde se llevan a cabo iniciativas de producción artesanal y distribución de bienes para modos de consumo alternativos. Además, coincide con la trama más irregular, con menos mobiliario y de frecuentación media.

El tercero y último, el más extendido, se caracteriza por su tendencia al incremento de locales dedicados a los servicios, por compartir espacio con los comercios tradicionales y la restauración, de trama regular, frecuentación media y accesible con diversos modos de transporte

Cabe mencionar que la falta de estudios que relacionen las variables de distinta naturaleza usadas en esta investigación es en sí misma una muestra del carácter exploratorio de la misma. En este sentido, nos parece importante señalar las limitaciones que presenta este estudio exploratorio, como por ejemplo no haber incluido la influencia del factor renta del suelo. Además, el análisis se ha llevado a cabo en calles que pertenecen a núcleos de población de tamaños demográficos distintos $y$, en este sentido, nos parece que en futuros trabajos se debería abordar la posible influencia de esta variable en los resultados. Finamente, para corroborar el método, sería interesante aplicar el modelo en núcleos de igual tamaño y complejidad territorial.

\section{Referencias bibliográficas}

AbELson, Elaine (1989). When ladies go a thieving: middle class shoplifters in the Victorian department store. Oxford: Oxford University Press.

Anselin, Luc (1992). Spatial Data Analysis with GIS: An Introduction to Application in the Social Sciences. Informe técnico, 10 de agosto de 1992. Santa Bárbara: University of California, National Center for Geographic Information and Analysis.

- (1996). «Local Indicators of Spatial Association (LISA)». Geographical Analysis, 27 (1), 93-115. 
Benson, Susan (1986). Counter cultures: saleswomen, managers and customers in American department stores. Urbana, IL: University of Illinois Press.

Boujaillat, Vincent y Rabailloud, Hervé (1989). «Les rues piétonnes lyonnaises ou le renforcement de la spécialisation commerciale au service de la centralité?». Pedestrian Streets in Lyon: The Strengthening of Retail Specialisation and an Increase of Centrality? Géocarrefour Année, 642, 99-110. Recuperado de <https://www. persee.fr/doc/geoca_0035-113x_1989_num_64_2_5676>.

Bratos, Martín (2013). «Business Improvement Districts: Una nueva forma de colaboración público-privada para la revitalización socioeconómica de áreas urbanas». Politica y Sociedad, 50 (1), 269-304. <https://doi.org/10.5209/rev_POSO.2013.v50.n1.38002>

Bros, Josep M.; Llobet, Josep y Mestres, Francesc (1995). «El comerç als centres urbans de la Regió Metropolitana de Barcelona». Revista Papers. Regió Metropolitana de Barcelona, 22, 61-68.

Casares, Javier (1995). «Factores de cambio en la distribución comercial». Derecho Privado y Constitución, 5, 67-84. Recuperado de <http://www.cepc.gob.es/publicaciones/revistas/revistaselectronicas?IDR=7\&IDN=379\&IDA=9937>.

Castellà, Anna; González, Dídac; Sardà, Jordi y Solé, Josep M. (7 de noviembre de 2018). Fris del comerç local [estudio principal]. Seminario en Escola Tècnica Superior d'Aquitectura de Reus. Campus Bellisens.

Cornide, Osvaldo (2020). Sin comercio no hay ciudad. Buenos Aires, 10/06/2020. Noticia. Circulares. CAME, Recuperado de <https://www.redcame.org.ar/contenidos/noticia/Sin-Comercio-No-Hay-Ciudad.430.html>.

Daniels, Peter W. (1985). Service industries: A geographical appraisal. Londres: Taylor $\&$ Francis.

Delclòs, Xavier y Miralles, Carme (2018). «Looking at Barcelona through Jane Jacobs's eyes: Mapping the basic conditions for urban vitality in a Mediterranean conurbation». Land Use Policy, 75, 505-517. <https://doi.org/10.1016/j.landusepol.2018.04.026>

Di Ciommo Floridea y Lucas Karen (2014). «Evaluating the equity effects of roadpricing in the European urban context - the Madrid metropolitan area». Applied Geography, 54, 74-82.

Duranton, Gilles y Overman, Henry G. (2005). "Testing for Localization Using Micro-Geographic Data». Review of Economic Studies, 72 (4), 1.077 1.106.

Elizagarate, Victoria y Zorrilla, Pilar (2004). «El comercio urbano como factor estratégico para la competitividad entre ciudades. Guipuzkoa: un caso de centro comercial abierto en red». Revista Distribución y Consumo, 78, 101-112. Recuperado de $<$ https://dialnet.unirioja.es/servlet/articulo?codigo $=1057544>$.

Elizagarate, Victoria; Mediano, Lucía y Domingo, Maite (2005). «Estrategias de marketing para la gestión público-privada del comercio de Guipúzcoa. Un modelo basado en el análisis clúster». Revista de Dirección y Administración de Empresas, $12,121-136$.

Entremont, Alban d' (1997). Geografía económica. Madrid: Cátedra.

Espinosa Seguí, Ana Isabel (2003). "Amenazas y nuevas estrategias del comercio de centro urbano. El caso de Alicante». Boletin de la Asociación de Geógrafos Españoles, 38, 153-174.

- (2007). El comercio como herramienta estructuradora del territorio: el caso de las ciudades alicantinas. Tesis de doctorado. Universidad de Alicante. 
Ferrer, Amador y Carrera, José María (1995). «L’espai i l'activitat comercial a Barcelona i la seva Área Metropolitana». Revista Papers. Regió Metropolitana de Barcelona, 22, 45-59.

Fujita, M. y Krugman, Paul (2004). "The New Economic Geography: Past, Present and the Future». Papers in Regional Science, 83 (1), 139-164.

Fujita, M. y Thisse, J. F. (2002). The Economics of Agglomeration. Cambridge: Cambridge University Press.

García, Miguel (2014). El deber de conservación y rehabilitación de los centros históricos urbanos. Repositorio Institucional Olavide. Tesis doctoral. Recuperado de $<$ http:// hdl.handle.net/10433/868>.

Garrocho, Carlos; Álvarez-lobato, José Antonio y Chávez, Tanoa (2012). «Aglomeración espacial de establecimientos comerciales en un centro tradicional de negocios. Un análisis basado en las funciones K». Economía Mexicana. Nueva época, xxi (1), 93-131.

GeHL, Jan (2006). EUA 09 - La humanización del espacio urbano. Trad.: Valcarce, MT (2020). Barcelona: Editorial Reverté. Visto en: Estudios Universitarios de Arquitectura, 9. Colección Jorge Sainz. Recuperado de <https://www.reverte.com/ media/reverte/files/sample-81190.pdfs.

GeHL, Jan (2010). Cities for people. Washington D. C.: Island Press.

Gehl, Jan y Svarre, Birgitte (2013). How to study public life. Washington D. C.: Island Press.

Guy, C. M. y David, G. (2004). «Measuring physical access to "healthy foods" in areas of social deprivation: A case study in Cardiff». International Journal of Consumer Studies, 28 (3), 222-234. <https://doi.org/10.1111/j.1470-6431.2003.00340.x>

Guzmán, Paloma; Pereira Roders, Ana y Colenbrander, Bernard (2017). «Measuring links between cultural heritage management and sustainable urban development: An overview of global monitoring tools». Cities, 60, 192-201. <https://doi.org/10.1016/j.cities.2016.09.005>

Haнm, Yeankyoung; Yoon, Heeyeun y Choi, Yunwon (2019). «The effect of built environments on the walking and shopping behaviors of pedestrians. A study with GPS experiment in Sinchon retail district in Seoul, South Korea». Cities, 89, 1-13. <https://doi.org/10.1016/j.cities.2019.01.020>

Isenberg, Alison (2004). Downtown America: A history of the place and the people who made it. Historical Studies of Urban America. Editado por Kathleen N. Conzen, Timothy J. Gilfoyle y James R. Grossman. Chicago: University of Chicago.

Jacobs, Jane (1961). The Death and Life of Great American Cities (1961). New York: Vintage Books

Johnson, Richard A. y Wichern, Dean W. (2007). Applied Multivariate Statistical Analysis. 6a edición. Upper Saddle River: Pearson Prentice Hall.

Krugman, Paul (1991). Geography and Trade. Cambridge: The MIT Press.

Lavadinho, Sonia (2014). "Dinámicas de proximidad en la ciudad: ideas para la transformación urbana». Ciudades, 17, 21-39. <https://doi.org/10.24197/ciudades.17.2014.21-39>

Londoño, M. Pilar (2015). Turismo gastronómico impulsor del comercio de proximidad. Barcelona: Editorial UOC.

Marshall, Alfred (1920). Principles of Economics: An Introductory Volume, 8a. Londres: MacMillan. 
Mulícek, Ondrej y Osman, Robert (2018). «Rhythm of urban retail landscapes: Shopping hours and the urban chronotopes». Moravian Geographical Reports, 26 (1), 2-13. <https://doi.org/10.2478/mgr-2018-0001>

Myınt, S. W. (2008). «An Exploration of Spatial Dispersion, Pattern, and Association of Socio-Economic Functional Units in an Urban System». Applied Geography, 28 (3), 168-188.

Northam, Raymond M. (1975). Urban Geography. USA: Library of Congress Cataloging in Publication Data.

Nosoohi, Iman y Zeinal-Hamadani, Ali (2011). "Urban Planning with the aid of Factor Analysis Approach: The Case of Isfahan Municipality». Theoretical and Empirical Researches in Urban Management, 6 (1), 56-69.

Nunes, P. A. L. D. (2002). "Using factor analysis to identify consumer preferences for the protection of a natural area in Portugal». European Journal of Operational Research, 140, 499-516.

O’Sullivan, Arthur (2007). Urban economics. Boston: McGraw-Hill.

PARIs, Mario (2013). «De los centros urbanos consolidados a los lugares de centralidad: una propuesta metodológica para su estudio». Ciudades, 16 (1), 47-69. Recuperado de <http://uvadoc.uva.es/handle/10324/10345>.

Park, Keunhyun; Ewing, Reid; Sabouri, Sadegh y Larsen, Jon (2018). «Street life and the built enviroment in an auto-oriented US region». Cities, 88, 243-251. <https://doi.org/10.1016/j.cities.2018.11.005>

Porter, Michael (1990). The competitive advantage of nations. USA: Free Press.

Pozueta, Julio; Lamiquiz, Francisco y Porto, Mateus (2009). La ciudad paseable. Recomendaciones para la consolidación de los peatones en el planeamiento, el diseño urbano y la arquitectura. Madrid: Ministerio de Fomento.

Rangel, Maritza Amelia (2009). "Indicadores de calidad de espacios públicos urbanos, para la vida ciudadana, en ciudades intermedias». En: Los pueblos americanos: cambios y continuidades. La construcción de lo propio en un mundo globalizado. Ciudad de México, del 19 al 24 de julio de 2009. 53 Congreso Internacional de Americanistas.

Rebollo, Arévalo (2003). «El papel de los mercados municipales en la vertebración de las tramas urbanas (1)». Distribución y Consumo, año 13 (69), 38-41. Recuperado de <https://dialnet.unirioja.es/servlet/articulo?codigo=633065>.

Ripley, B. D. (1976). «The Second-Order Analysis of Stationary Point process». Journal of Applied Probability, 13 (1), 255-266.

Rivas, Juan Luis (2007). «Dificultades del urbanismo commercial en el Plan General de Equipamientos Comercial de Castilla y León». Ciudades, 10, 109-142.

Rosenthal, Stuart S. y Strange, William C. (2004). «Evidence on the Nature and Sources of Agglomeration Economies». En: Henderson, J. V. y Thisse, J. (eds.). Handbook of Regional and Urban Economics, 4. Ámsterdam. Recuperado de $<$ http://www.econ.brown.edu/faculty/henderson/willandstuart.pdf $>$.

Sanz, Alfonzo (1996). Calmar el tráfico. Madrid: Ministerio de Obras Públicas, Transportes y Medio Ambiente.

Sassano, Silvana (2015). «Imagen, localización y evolución de los centros comerciales en Argentina. Un estudio de caso (Buenos Aires)». Documents d'Anàlisi Geogràfica, 61 (2), 409-432. <https://doi.org/10.5565/rev/dag.238>

Segado-VÁzquez, Francisco Enrique y Espinosa-Muñoz, Víctor Manuel (2015). «La ciudad herida. Siete ejemplos paradigmáticos de rehabilitación urbana en la 
segunda mitad del siglo xx». EURE, Revista Latinoamericana de Estudios Urbanos Regionales, 41 (123), 103-129.

Serra, Pere; Vera, Ana; Tulla, Antoni Francesc y Salvati, Luca (2014). «Beyond ruban-rural dichotomy explorin socioeconòmic and land-use processes of change in Spain (1991-2011)». Applied Geography, 55, 71-81.

Sevtsuk, Andres (2020). Street Commerce. Creating Vibrant Urban. Filadelfia: University of Pennsylvania Press.

Simó, Meritxell; Casellas, Antònia y Avellaneda, Pau (2018). "Comercio minorista y peatonalización: evolución y adaptación en la ciudad costera de Malgrat de Mar (Barcelona)». Revista: Anales de Geografia de la Universidad Complutense, 38 (1), 219-238. <https://doi.org/10.5209/AGUC.60476>

Somoza-Medina, Xosé y López-González, Alejandro (2017). "Crisis, comercio y ciudad. Cambios en la actividad comercial y el consumo en España». Revista de Estudios Andaluces, 34 (1), 93-320. $<$ http://dx.doi.org/10.12795/rea.2017.i34.10>

Stoica, V. I.; Tulla, A. F.; Zamfir, D. y Petrisor, A. (2020). «Exploring the Urban Strength of Small Towns in Romania». Social Indicators Research, 152, 843-875. <https://doi.org/10.1007/s11205-020-02465-x>

Talavera-García, Rubén; Soria-Lara, Julio y Valenzuela-Montes, Luis Miguel (2014). «La calidad peatonal como método para evaluar entornos de movilidad urbana». Documents d'Anàlisi Geogràfica, 60 (1), 161-187. <https://doi.org/10.5565/rev/dag.55>

Venturini, Edgadro J. (1992). "Centros urbanos, patrimonio e identidad ciudadana en el mundo de la globalización». Estudios, 15, 157-168.

Zárate, M. Antonio (2006). «Medio siglo de cambios en los centros urbanos españoles». Estudios Geográficos, 67 (260), 283-315. <https://doi.org/10.3989/egeogr.2006.i260.51>

Zhang, Yuyang; Yang, Bowen; ZHAng, Mengcai; Zhang, Gong; Song, Shanshan y LING, Qi (2019). "Exploring Location Pattern of Commercial Stores in Shichahai, Beijing from a Street Centrality Perspective». Chin.Geogra.Sci, 29 (3), 503-516. <https://doi.org/10.1007/s11769-019-1045-z> 
